\title{
MULTIPLE PERIODIC SOLUTIONS FOR AUTONOMOUS CONSERVATIVE SYSTEMS
}

\author{
Silvia Cingolani - Monica Lazzo
}

\begin{abstract}
We consider an autonomous conservative second order system defined by a potential which admits a connected set $\Gamma$ of critical points at level zero. We prove the existence of multiple periodic solutions of large period which are located near $\Gamma$.
\end{abstract}

\section{Introduction and statement of the results}

In the present paper, we deal with an autonomous conservative second order Hamiltonian system defined by a potential $V$ which admits a connected set $\Gamma$ of critical points at level zero. We are interested in finding periodic orbits with large period $T$, namely $T$-periodic solutions to

$$
\ddot{x}(t)=V^{\prime}(x(t)) \text { in } \mathbb{R},
$$

where $V \in C^{1}\left(\mathbb{R}^{2}, \mathbb{R}\right)$ and $V^{\prime}$ denotes the gradient of $V$.

Plainly, any point in $\Gamma$ is a trivial solution to $(\mathrm{P})$; as a consequence, looking for nontrivial solutions to $(\mathrm{P})$ requires avoiding the constant solutions at level zero.

When the problem has a suitable symmetry, namely $V$ is even, a $\mathbb{Z}_{2}$-version of Ljusternik-Schnirelman theory can be applied. If $\Gamma$ is a circle or an annulus in

1991 Mathematics Subject Classification. 34C25, 34B15.

Key words and phrases. Periodic solutions, singular potentials, winding number.

Supported by M.U.R.S.T. (40\% and 60\% funds) and E.E.C., Program Human Capital Mobility (Contract ERBCHRXCT 940494). 
the plane, it has a finite $\mathbb{Z}_{2}$-index; as far as large period solutions are concerned, this allows avoiding trivial solutions. We refer to Remark 5.2 for some details on such an approach.

In the general case, $(\mathrm{P})$ has still an intrinsic symmetry, i.e. it is $S^{1}$-invariant. Nevertheless, in the situations we are interested in the $S^{1}$-index of $\Gamma$ (see [2]) is not finite, hence an $S^{1}$-version of Ljusternik-Schnirelman theory cannot be successfully applied.

In order to prove existence of multiple nontrivial periodic solutions of large period to $(\mathrm{P})$, without evenness assumptions on $V$, our idea consists in regarding (P) as a problem with a singular potential. Indeed, a simple change of variable proves that $T$-periodic solutions for $(\mathrm{P})$ correspond to 1 -periodic solutions for

$$
\ddot{x}(t)=T^{2} V^{\prime}(x(t)) \quad \text { in } \mathbb{R} .
$$

Since, for any point $\xi \in \mathbb{R}^{2}$ with $V(\xi)>0$, the nonlinear term $T^{2} V(\xi)$ becomes larger and larger as $T$ increases, we can say that $\left(\mathrm{P}_{T}\right)$ looks like a problem with singular potential. As a consequence, we obtain our multiplicity result by a standard minimization argument in a class of functions which wind around some point $\xi$ with $V(\xi)>0$. Let us recall that existence and multiplicity of periodic solutions with singular potential have been extensively investigated by many authors; for instance, see [1], [4], [9] for strong-force potential, [7], [12], [13] for weak-force potential and references therein.

Before stating our main results, we need some notations and definitions. We say that $\Gamma \subset \mathbb{R}^{2}$ is an admissible set if it is bounded and its complementary $\Gamma^{c}$ has exactly two connected components. Remark that, by its very definition, an admissible set is not simply connected in the plane.

For any admissible set $\Gamma$ we denote by $\mathcal{B}$ and $\mathcal{U}$, respectively, the bounded and the unbounded connected component of $\Gamma^{c}$. Moreover, for any $r>0$ we set $\mathcal{B}_{r}=\{\xi \in \mathcal{B}: \operatorname{dist}(\xi, \Gamma) \leq r\}, \mathcal{U}_{r}=\{\xi \in \mathcal{U}: \operatorname{dist}(\xi, \Gamma) \leq r\}$.

Theorem 1.1. Let $\Gamma$ be an admissible set such that $V \equiv 0$ on $\Gamma$. Assume that for some $r>0$

$$
V(x)>0 \quad \text { for any } x \in \mathcal{B}_{r} \cup \mathcal{U}_{r} .
$$

Moreover, assume that $\Gamma$ contains the support of a noncontractible closed curve of class $H^{1}$. Then, for any $k \in \mathbb{N}$ there exists $T_{k}>0$ such that $(\mathrm{P})$ has at least $k$ distinct nontrivial $T$-periodic solutions, for any $T>T_{k}$.

As a model situation, one can consider $V\left(x_{1}, x_{2}\right)=\left(|x|^{2}-1\right)^{2} e^{x_{1}}, x=$ $\left(x_{1}, x_{2}\right)$; in this case, $\Gamma=S^{1}$ and all the assumptions in Theorem 1.1 are fulfilled for any $0<r<1$.

We point out that in Theorem 1.1 no assumptions on the behaviour of the potential at infinity are required. In our approach, the existence of nontrivial 
$T$-periodic solutions of $(\mathrm{P})$ depends only on the behaviour of the potential in a neighbourhood of $\Gamma$.

As concerns the hypothesis about the existence of a noncontractible closed curve of class $H^{1}$ with support in $\Gamma$, let us note that it is satisfied in many situations. For example, it holds if $\Gamma$ is diffeomorphic to $S^{1}$ or to an annulus in the plane; more in general, if there exists a piecewise differentiable homeomorphism between $S^{1}$ or an annulus and $\Gamma$. Moreover such an hypothesis is plainly satisfied if $\overline{\mathcal{B}} \cap \overline{\mathcal{U}}=\emptyset$; roughly speaking, this means that $\Gamma$ is a "thick" set. The existence of such a curve in $\Gamma$ is needed for technical reasons; as it will be clear in Section 3, we use it to obtain a uniform control on the minima of the action functional associated to $\left(\mathrm{P}_{T}\right)$. In this sense, such a curve can be considered as a "comparison term".

In the next theorem we consider a particular case, namely we assume that the exterior boundary of $\Gamma$ (that is, $\Gamma \cap \overline{\mathcal{U}}$ ) is a circle in the plane. Such a geometrical hypothesis has two main consequences. Firstly, it guarantees the existence of a comparison term, in the sense of the previous remark. Secondly, it allows an easy modification of $V$ in $\mathcal{U}$ such that the solutions of the modified equation still solve $\left(\mathrm{P}_{T}\right)$. As a result, the behaviour of $V$ in the unbounded component of $\Gamma^{c}$ is not influent, and assumption (1.1) in Theorem 1.1 can be weakened.

Theorem 1.2. Let $\Gamma$ be an admissible set such that $V \equiv V^{\prime} \equiv 0$ on $\Gamma$. Assume that $\Gamma \cap \overline{\mathcal{U}}$ is a circle in the plane and

$$
V(x)>0 \quad \text { for any } x \in \mathcal{B}_{r} .
$$

Then, for any $k \in \mathbb{N}$ there exists $T_{k}>0$ such that $(\mathrm{P})$ has at least $k$ distinct nontrivial T-periodic solutions, for any $T>T_{k}$.

Let us remark that in Theorem 1.1 the condition $V^{\prime} \equiv 0$ is obviously satisfied. In Theorem 1.2, $V$ can exhibit any behaviour outside $\Gamma$, provided it is flat on $\Gamma$.

Finally, in this paper we describe the qualitative behaviour of $T$-periodic solutions to $(\mathrm{P})$ as the period becomes larger and larger. Precisely, we show that if $x_{T}$ is any solution found via Theorem 1.1 or 1.2 , then as $T \rightarrow \infty x_{T}$ approaches a curve $x_{0}$, such that $x_{0}(t) \in \Gamma$ for any $t$ (we refer to Remark 5.1 for details). As a consequence, the long period periodic solutions of $(\mathrm{P})$ are located near $\Gamma$. Since each point in $\Gamma$ is a constant periodic solution of $(\mathrm{P})$, in some sense we can say that we obtain a "Birkhoff-Lewis" type result (see the classical paper [5] and also [3] and references therein).

\section{Variational setting and preliminaries}

Let us introduce some notations. Let $H$ be the Sobolev space of the absolutely continuous 1-periodic curves in $\mathbb{R}^{2}$ with square integrable derivative, 
endowed with the usual norm $\|\cdot\|$. The action functional associated with problem $\left(\mathrm{P}_{T}\right)$ is

$$
f_{T}(x)=\frac{1}{2} \int_{0}^{1}|\dot{x}(t)|^{2} d t+T^{2} \int_{0}^{1} V(x(t)) d t, \quad x \in H .
$$

REmark 2.1. For any $T>0, f_{T}$ is weakly lower semicontinuous in $H$, as it is the sum of a weakly lower semicontinuous functional (the quadratic term) and a weakly continuous one. Indeed, $x_{n} \rightarrow x$ in $H$ implies $x_{n} \rightarrow x$ uniformly and the continuity of $V$ yields $\int_{0}^{1} V\left(x_{n}(t)\right) d t \rightarrow \int_{0}^{1} V(x(t)) d t$ as $n \rightarrow \infty$.

We aim to find critical points of $f_{T}$ as minima in suitable homotopy classes and to this end we shall adapt the arguments in [6].

Let $r>0$ be fixed as in Theorem 1.1. Let $0<\rho<r$ and define

$$
\Lambda=\{x \in H: \operatorname{dist}(x(t), \Gamma)<\rho \text { for all } t \in[0,1]\} ;
$$

plainly, $\Lambda$ is an open subset of $H$ whose boundary is $\partial \Lambda=\{x \in H$ : exists $t \in$ $[0,1]$ s.t. $\operatorname{dist}(x(t), \Gamma)=\rho\}$. Any $x \in \Lambda$ is a closed curve in the plane that does not cross any point in the set $\mathcal{B} \backslash \mathcal{B}_{r}$ (cf. Section 1 for notations); therefore, we can consider the winding number of $x$ around any such point, which we shall denote by $\operatorname{Ind}(x)$. Finally, for any $q \in \mathbb{Z}$ define

$$
\Lambda_{q}=\{x \in \Lambda: \operatorname{Ind}(x)=q\} .
$$

Let $\gamma \in H$ be a noncontractible closed curve such that $\gamma([0,1]) \subset \Gamma$; the existence of such a curve is one of the assumptions in Theorem 1.1. With no restrictions, we can suppose $\operatorname{Ind}\left(\gamma_{\mid[0,1]}\right)=1$. Plainly, the curve $\gamma_{q}(t)=\gamma(q t), t \in[0,1]$, belongs to $\Lambda_{q}$. The main properties of $\Lambda_{q}$ are collected in the following lemma.

Lemma 2.2. For any $q \in \mathbb{Z}, \Lambda_{q}$ is an open connected subset of $\Lambda$. Furthermore, $\Lambda_{q}$ is weakly closed in $\Lambda$.

Proof. Let $q \in \mathbb{Z}$. The first assertions follow from the elementary properties of the winding number. To prove the last statement, let $\left\{x_{n}\right\} \subset \Lambda_{q}$ and $x \in \Lambda$ be such that $x_{n}$ weakly converges to $x$ in $\Lambda$. Choose $\xi \in \mathcal{B} \backslash \mathcal{B}_{r}$; plainly, $|x(t)-\xi|>$ $r-\rho$ for any $t$. On the other hand, as $x_{n}$ converges to $x$ uniformly in $[0,1]$, for $n$ large we have $\left|x_{n}(t)-x(t)\right|<r-\rho$ for any $t$; thus $\operatorname{Ind}\left(x_{n}\right)=\operatorname{Ind}(x)$ for $n$ large, whence $x \in \Lambda_{q}$.

The next result is analogous to the well known Gordon Lemma [8], concerning singular strong-force potential.

Lemma 2.3. For any $M>0$ there exists $\bar{T}>0$ such that the sublevel set $f_{T}^{M}=\left\{x \in \Lambda: f_{T}(x) \leq M\right\}$ is weakly sequentially compact, for any $T \geq \bar{T}$.

Proof. By contradiction, assume that there exist $M>0, T_{k} \rightarrow \infty$ and a sequence $\left\{x_{n, k}\right\} \subset f_{T_{k}}^{M}$ which has no subsequences weakly converging in $f_{T_{k}}^{M}$. As 
$\left\{x_{n, k}\right\}$ is bounded in $H$, it certainly has a subsequence, still denoted by $\left\{x_{n, k}\right\}$, which converges to some $x_{k}$ weakly in $H$, strongly in $L^{2}$ and uniformly in $[0,1]$. By weak lower semicontinuity, $f_{T_{k}}\left(x_{n, k}\right) \leq M$, so necessarily $x_{k} \in \partial \Lambda$. As a result, there exists $t_{k} \in[0,1]$ such that $\operatorname{dist}\left(x_{k}\left(t_{k}\right), \Gamma\right)=\rho$. Let $0<\rho_{1}<\rho$ be such that $0<\alpha \leq V(\xi)$ if $\rho_{1} \leq \operatorname{dist}(\xi, \Gamma) \leq \rho$. For any $t \in[0,1]$ we have

$$
\left|x_{k}(t)-x_{k}\left(t_{k}\right)\right| \leq c\left|t-t_{k}\right|^{1 / 2}\left\|\dot{x}_{k}\right\|_{2} .
$$

Since $\left\|x_{k}\right\|^{2} \leq \liminf _{n \rightarrow \infty}\left\|x_{n, k}\right\|^{2} \leq\left\|x_{k}\right\|_{2}^{2}+2 M$, we get $\left\|\dot{x}_{k}\right\|_{2}^{2} \leq 2 M$. Then (2.1) implies $\rho_{1} \leq \operatorname{dist}\left(x_{k}(t), \Gamma\right) \leq \rho$ for any $t \in\left[t_{k}-\delta, t_{k}+\delta\right]$, for some $\delta>0$. Hence

$$
\begin{aligned}
M & \geq T_{k}^{2} \int_{0}^{1} V\left(x_{k}(t)\right) d t \geq T_{k}^{2} \int_{t_{k}-\delta}^{t_{k}+\delta} V\left(x_{k}(t)\right) d t \\
& \geq 2 \delta T_{k}^{2} \min _{\left[t_{k}-\delta, t_{k}+\delta\right]} V\left(x_{k}(t)\right) \geq \delta \alpha T_{k}^{2}
\end{aligned}
$$

which yields a contradiction as $k \rightarrow \infty$.

\section{Proof of Theorem 1.1}

The curve $\gamma$ we introduced in Section 2 plays a relevant role in the following result.

Proposition 3.1. For any $q \in \mathbb{Z}$, there exists $T_{q}>0$ such that $f_{T}$ attains its infimum in $\Lambda_{q}$, for any $T \geq T_{q}$.

Proof. Let $q \in \mathbb{Z}$; as in Section 2, let $\gamma_{q}(t)=\gamma(q t)$. Let $M_{q} \equiv f_{T}\left(\gamma_{q}\right)=$ $\left(q^{2} / 2\right) \int_{0}^{1}|\dot{\gamma}(t)|^{2} d t$. Let $\bar{T}_{q}>0$ be associated with $2 M_{q}>0$ as in Lemma 2.3. Let $T \geq \bar{T}_{q}$ and let $\left\{x_{n, T}\right\} \subset \Lambda_{q}$ be a minimizing sequence, namely $f_{T}\left(x_{n, T}\right) \rightarrow$ $\inf _{\Lambda_{q}} f_{T}$ as $n \rightarrow \infty$. For $n$ sufficiently large $f_{T}\left(x_{n, T}\right) \leq 2 M_{q}$. By Lemmas 2.2 and 2.3, $\left\{x_{n, T}\right\}$ weakly converges to some $x_{T} \in \Lambda_{q}$. Finally, Remark 2.1 gives $f_{T}\left(x_{T}\right) \leq \liminf _{n \rightarrow \infty} f_{T}\left(x_{n, T}\right)=\inf _{\Lambda_{q}} f_{T}$, whence $x_{T}$ is a minimum point for $f_{T}$ in $\Lambda_{q}$.

Proof of Theorem 1.1. Let $k \in \mathbb{N}$ and $0<q_{1}<\ldots<q_{k}$. By Proposition 3.1 , for any $j=1, \ldots, k$ there exists $T_{q_{j}}>0$ such that for any $T \geq$ $T_{q_{j}}$ the functional $f_{T}$ has a critical point $x_{q_{j}}$ with $\operatorname{Ind}\left(x_{q_{j}}\right)=q_{j}$. Let $\bar{T}=$ $\max \left\{T_{q_{1}}, \ldots, T_{q_{k}}\right\}$. Then, for any $T \geq \bar{T}$ the functional $f_{T}$ has $k$ critical points $x_{q_{j}}, j=1, \ldots, k$ with $\operatorname{Ind}\left(x_{q_{j}}\right)=q_{j}$. Therefore there exist $k$ nontrivial distinct periodic solutions of $\left(\mathrm{P}_{T}\right)$, for any $T \geq \bar{T}$.

\section{Proof of Theorem 1.2}

Without restrictions, in Theorem 1.2 we can assume $\Gamma \cap \overline{\mathcal{U}}=S^{1}$. Before proving the theorem, some remarks are in order. In the proof of Theorem 1.1, the key point consists in proving that, for large $T$, the $H^{1}$-weak limit $x$ of any 
sequence $\left\{x_{n}\right\}$ in $\Lambda$, satisfying $f_{T}\left(x_{n}\right) \leq$ const, is again in $\Lambda$ (this is substantially the meaning of Lemma 2.3). Roughly speaking, $x \in \partial \Lambda$ if for some $t$ either $x(t) \in \mathcal{B}$ and $\operatorname{dist}(x(t), \Gamma)=\rho$ or $x(t) \in \mathcal{U}$ and the same equality holds. In order to prove that none of these cases can occur, we take advantage of the behaviour of $V$ around $\Gamma$, as prescribed in (1.1). In Theorem 1.2, the behaviour of $V$ around $\Gamma$ is prescribed only on the bounded component of $\Gamma^{c}$. Therefore, we are no more able to prevent the case $x(t) \in \mathcal{U}$ and $\operatorname{dist}(x(t), \Gamma)=\rho$. To overcome such a difficulty, we look for critical points of $f_{T}$ in a set different from $\Lambda$. Precisely, let $r>0$ be fixed as in Theorem 1.2, let $0<\rho<r$ and $\widetilde{\Gamma}=\{\xi \in \mathcal{B}: \operatorname{dist}(\xi, \Gamma)<\rho\} \cup \Gamma \cup \mathcal{U}$; we define

$$
\widetilde{\Lambda}=\{x \in H: x(t) \in \widetilde{\Gamma} \text { for all } t \in[0,1]\} .
$$

Again, $\widetilde{\Lambda}$ is an open subset of $H$ whose boundary is $\partial \widetilde{\Lambda}=\{x \in H$ : exists $t \in$ $[0,1]$ s.t. $x(t) \in \mathcal{B}$, $\operatorname{dist}(x(t), \Gamma)=\rho\}$. Exactly as before, we can define $\operatorname{Ind}(x)$ and consider $\widetilde{\Lambda}_{q}=\{x \in \widetilde{\Lambda}: \operatorname{Ind}(x)=q\}$, for $q \in \mathbb{Z}$. Plainly, Lemma 2.2 still holds for any $\widetilde{\Lambda}_{q}$; let us just point out that every such set is nonempty because it contains the curve $\exp (i 2 \pi q t), t \in[0,1]$.

As concerns Lemma 2.3, in the present situation a crucial ingredient is missing. Indeed, in the proof of Lemma 2.3 we use the fact that any sequence $\left\{x_{n}\right\} \subset \Lambda$ satisfying $f_{T}\left(x_{n}\right) \leq C$, for some $C>0$, is bounded in $H$. This follows from the very definition of the action functional and the fact that $\Lambda$ is bounded in $L^{\infty}$, hence in $L^{2}$. This last assertion does not hold for $\widetilde{\Lambda}$. To get round this difficulty, we replace $V$ by a coercive potential in such a way that the critical points of the modified action functional are still critical points of $f_{T}$. At this point, the assumption $\Gamma \cap \overline{\mathcal{U}}=S^{1}$ is used to construct the modified potential; a similar modification is used in [10].

Lemma 4.1. Let $\widetilde{V} \in C^{1}\left(\mathbb{R}^{2}, \mathbb{R}_{+}\right)$be such that $\widetilde{V}(x)=V(x)$ for $|x| \leq 1$ and $\left(\widetilde{V}^{\prime}(x), x\right)>0$ for any $|x|>1$. For any $x \in H$ let

$$
\widetilde{f}_{T}(x)=\frac{1}{2} \int_{0}^{1}|\dot{x}(t)|^{2} d t+T^{2} \int_{0}^{1} \widetilde{V}(x(t)) d t .
$$

Then any $x \in H$ critical point for $\widetilde{f}_{T}$ is a critical point for $f_{T}$.

Proof. Let $x \in H$ be a critical point for $\tilde{f}_{T}$ and let us define

$$
x_{0}(t)= \begin{cases}x(t) & \text { if }|x(t)| \leq 1, \\ x(t) /|x(t)| & \text { if }|x(t)|>1 .\end{cases}
$$

We have

$$
0=\left\langle\widetilde{f}_{T}^{\prime}(x), x-x_{0}\right\rangle=\int_{0}^{1}\left(\dot{x}, \dot{x}-\dot{x}_{0}\right) d t+T^{2} \int_{0}^{1}\left(\widetilde{V}^{\prime}(x), x-x_{0}\right) d t .
$$


Furthermore,

$$
\begin{aligned}
\int_{0}^{1}\left(\dot{x}, \dot{x}-\dot{x}_{0}\right) d t & =\int_{|x(t)|>1}\left(\dot{x}, \dot{x}-\dot{x}_{0}\right) d t \\
& =\int_{|x(t)|>1}\left(\dot{x}, \dot{x}-\frac{\dot{x}}{|x|}+\frac{(\dot{x}, x)}{|x|^{3}} x\right) d t \\
& =\int_{|x(t)|>1}\left(\left(1-\frac{1}{|x|}\right)|\dot{x}|^{2}+\frac{(\dot{x}, x)^{2}}{|x|^{3}}\right) d t \geq 0 .
\end{aligned}
$$

If $x \not \equiv x_{0}$, then

$$
\int_{0}^{1}\left(\widetilde{V}^{\prime}(x), x-x_{0}\right) d t=\int_{|x(t)|>1}\left(1-\frac{1}{|x|}\right)\left(\widetilde{V}^{\prime}(x), x\right) d t>0 ;
$$

the last inequality, (4.1) and (4.2) give a contradiction.

It is very easy to give examples of $C^{1}$ maps $\widetilde{V}$ satisfying the hypotheses of Lemma 4.1. For instance, one can consider

$$
\widetilde{V}(x)= \begin{cases}V(x) & \text { for }|x| \leq 1, \\ 2|x|^{3}-3|x|^{2}+1 & \text { for }|x|>1,\end{cases}
$$

note that $\widetilde{V}$ is $C^{1}$ because $V \equiv V^{\prime} \equiv 0$ on $S^{1}$.

By Lemma 4.1, we can now assume $V$ coercive at infinity; moreover, any critical point $x_{T}$ of the modified functional satisfies $\left\|x_{T}\right\|_{\infty} \leq 1$. At this point, with minor changes in the proof of Lemma 2.3 we obtain

Lemma 4.2. For any $M>0$ there exists $\bar{T}>0$ such that the sublevel set $\left\{x \in \widetilde{\Lambda}: f_{T}(x) \leq M\right\}$ is weakly sequentially compact, for any $T \geq \bar{T}$.

As a result, we can easily prove

Proposition 4.3. For any $q \in \mathbb{Z}$, there exists $T_{q}>0$ such that $f_{T}$ attains its infimum in $\widetilde{\Lambda}_{q}$, for any $T \geq T_{q}$.

Let us point out that in the proof of Proposition 4.3 the natural comparison term $\exp (i 2 \pi q t), t \in[0,1]$, can be used. Finally, arguing exactly as in the proof of Theorem 1.1 we can conclude the proof of Theorem 1.2.

\section{Final remarks}

In the following remark we describe the qualitative behaviour of the $T$ periodic solutions found via Theorem 1.1 and 1.2 as the period increases.

Remark 5.1. Let $q \in \mathbb{Z}$ and $T_{q}>0$ be as in Proposition 3.1 (respectively, in Proposition 4.3). For any $T \geq T_{q}$, let $x_{T}$ be a minimum point for $f_{T}$ in $\Lambda_{q}$ (respectively in $\widetilde{\Lambda}_{q}$ ). As the minimum level of the action functional is bounded by a positive constant $C=C(q)$ which depends on $q$ and is independent of $T$, 
the same holds for $\left\|x_{T}\right\|$. Then there exists a function $x_{0}$ such that $x_{T} \rightarrow x_{0}$, as $T \rightarrow \infty$, weakly in $H$ and uniformly in $[0,1]$. We claim that $x_{0}(t) \in \Gamma$ for any $t \in[0,1]$. By contradiction, assume $\operatorname{dist}\left(x_{0}(t), \Gamma\right)>\beta>0$ for any $t$ in some interval $\left[t_{1}, t_{2}\right] \subset[0,1]$. Therefore, for $T$ large, $\operatorname{dist}\left(x_{T}(t), \Gamma\right) \geq \beta$ in $\left[t_{1}, t_{2}\right]$; on the other hand, by construction, $\operatorname{dist}\left(x_{T}(t), \Gamma\right) \leq \rho$. If we set $m=\min \left\{V(\xi): \xi \in \mathbb{R}^{2}, \beta \leq \operatorname{dist}(\xi, \Gamma) \leq \rho\right\}$ (respectively $m=\min \{V(\xi):|\xi| \leq$ $1, \beta \leq \operatorname{dist}(\xi, \Gamma) \leq \rho\})$, then $m>0$ and

$$
C \geq T^{2} \int_{0}^{1} V\left(x_{T}(t)\right) d t \geq T^{2} \int_{t_{1}}^{t_{2}} V\left(x_{T}(t)\right) d t \geq m T^{2}\left(t_{2}-t_{1}\right)
$$

for any $T$ large, a contradiction.

REMARK 5.2. As we noted in the Introduction, when $V$ has a suitable symmetry a $\mathbb{Z}_{2}$-version of Ljusternik-Schnirelman theory can be easily applied to find multiple solutions to $\left(\mathrm{P}_{T}\right)$. Let us give an example, referring to $[2,11]$, where similar situations are dealt with, for some details. For the sake of simplicity, assume that $V \geq 0$ in $\mathbb{R}^{2}$ and $V(\xi)=0$ iff $|\xi|=1$. Assume $V(-\xi)=V(\xi)$ for any $|\xi| \leq 1$. Furthermore, suppose that $V$ is of class $C^{2}$ around the origin, $\xi=0$ is a local maximum of $V$ and the Hessian matrix $V^{\prime \prime}(0)$ has at least one nonzero eigenvalue. Let us explicitly remark that the assumptions on $V$ involve only the unit disc; indeed, as shown in Section 4 we can modify $V$ outside the unit circle in such a way that the action functional $f_{T}$ satisfies Palais-Smale condition. Let $\Sigma$ be the set of closed, symmetric subset of $H$ which do not contain 0 ; for $A \in \Sigma$, let $\gamma(A)$ be the $\mathbb{Z}_{2}$-genus of $A$. Standard theory implies that the inf-max levels defined by

$$
c_{q}=\inf _{\substack{A \in \Sigma \\ \gamma(A) \geq q}} \max _{x \in A} f_{T}(x), \quad q \geq 1,
$$

are critical levels for $f_{T}$. To distinguish $c_{q}$ from the trivial critical levels 0 and $f_{T}(0)$, some remarks on the genus of suitable sets are in order. Firstly, observe that $c_{q}>0$ for any $q \geq 3$; indeed, if $0=c_{1}=\ldots=c_{l}$ for some $l$, then $l$ is less than the genus of the set of critical points of $f_{T}$ at level 0 , that is $\gamma\left(S^{1}\right)=2$. Secondly, let $L_{T}$ be the self-adjoint realization in $L^{2}(0,1)$ of the operator $x \mapsto-\ddot{x}+T^{2} V^{\prime \prime}(0) x$, with 1-periodicity conditions, and $Z_{T}$ be the subspace of $H$ spanned by the nonconstant eigenfunctions of $L_{T}$ corresponding to strictly negative eigenvalues. It is easy to see that there exists $\delta(T)>0$ such that $\sup \left\{f_{T}(x): x \in Z_{T} \cap \partial B_{\delta(T)}\right\}<T^{2} V(0)=f_{T}(0)$. Remark that $q_{T} \equiv$ $\gamma\left(Z_{T} \cap \partial B_{\delta(T)}\right)=\operatorname{dim} Z_{T}$; as a consequence, $q_{T}$ increases to $+\infty$ as $T$ increases to $+\infty$. Therefore, for any fixed integer $k$, there exists $T_{k}>0$ such that for any $T>T_{k}$ we have $0<c_{3} \leq c_{2+k} \leq c_{q_{T}}<f_{T}(0)$, which implies that $\left(\mathrm{P}_{T}\right)$ has at least $k$ nontrivial periodic solutions. Let us finally remark that this result still holds if the plane is replaced by $\mathbb{R}^{m}, m \geq 2$. 


\section{REFERENCES}

[1] A. Ambrosetti and V. Coti Zelati, Periodic solutions of singular dynamical systems, Nato Adv. Sci. Inst. Ser., Periodic Solutions of Hamiltonian Systems and Related Topics (P.H: Rabinowitz et al., eds.), vol. 209, Reidel, Dordrecht, 1987, pp. 1-10.

[2] V. Benci, A geometrical index for the group $S^{1}$ and some applications to the study of periodic solutions of ordinary differential equations, Comm. Pure Appl. Math. 34 (1981), 393-432.

[3] V. Benci And D. Fortunato, A "Birkhoff-Lewis" type result for non autonomous differential equations, Proc. ELAM VIII Lecture Notes in Mathematics 1324 (1988), Springer-Verlag, 85-96.

[4] V. Benci And F. Giannoni, Periodic solutions of prescribed energy for a class of Hamiltonian systems with singular potential, J. Differential Equations 157 (1991), 568576.

[5] G. D. BIRkhofF AND D. C. Lewis, On the periodic motions near a given periodic motion of a dynamical system, Ann. Mat. Pura Appl. 12 (1933), 117-133.

[6] S. Cingolani, M. Lazzo and J. F. Padial, Multiple radial solutions for a class of elliptic systems with singular nonlinearities, Ann. Mat. Pura Appl. IV 175 (1998), 365-373.

[7] V. Coti Zelati, Periodic solutions for a class of planar singular dynamical systems, J. Math. Pures Appl. 68 (1989), 109-119.

[8] W.B. Gordon, Conservative dynamical systems involving strong forces, Trans. Amer. Math. Soc. 204 (1975), 113-135.

[9] C. Greco, Periodic solutions of a class of singular Hamiltonian systems, Nonlinear Anal. 12 (1988), 259-269.

[10] D. PASsAseo, Multiplicity of critical points for some functionals related to the minimal surfaces problem, Calc. Var. Partial Differential Equations 6 (1998), 105-121.

[11] P. Rabinowitz, Periodic and heteroclinic orbits for a periodic hamiltonian system, Ann. Inst. H. Poincaré Anal. Non Linéaire 6 (1989), 331-346.

[12] E. SERrA AND S. TERrACINI, Noncollision solutions to some singular minimization problems with Keplerian-like potentials, Nonlinear Anal. 22 (1994), 45-62.

[13] S. Terracini, An homotopical index and multiplicity of periodic solutions to dynamical systems with singular potential, J. Differential Equations 98 (1992), 197-225.

Manuscript received July 20, 1999

Silvia Cingolani and Monica Lazzo

Dipartimento di Matematica

via Orabona 4

70125 Bari, ITALY

E-mail address: cingolan@pascal.dm.uniba.it, lazzo@pascal.dm.uniba.it

TMNA : Volume $14-1999-\mathrm{N}^{\circ} 1$ 